\title{
Hemodynamic Analysis of Pediatric Septic Shock and Cardiogenic Shock Using Transpulmonary Thermodilution
}

\author{
En-Pei Lee, ${ }^{1,2}$ Shao-Hsuan Hsia, ,,2 Jainn-Jim Lin, ${ }^{1,2}$ Oi-Wa Chan, ${ }^{1,2}$ Jung Lee, \\ Chia-Ying Lin, ${ }^{1,2}$ and Han-Ping $\mathrm{Wu}^{2,3}$ \\ ${ }^{1}$ Division of Pediatric Critical Care Medicine, Department of Pediatrics, Chang Gung Memorial Hospital, Linko, \\ Kweishan, Taoyuan, Taiwan \\ ${ }^{2}$ College of Medicine, Chang Gung University, Taoyuan, Taiwan \\ ${ }^{3}$ Division of Pediatric General Medicine, Department of Pediatrics, Chang Gung Memorial Hospital, Linko, \\ Kweishan, Taoyuan, Taiwan \\ Correspondence should be addressed to Han-Ping Wu; arthur1226@gmail.com
}

Received 13 January 2017; Revised 21 February 2017; Accepted 28 February 2017; Published 16 March 2017

Academic Editor: Kazuyoshi Suenari

Copyright (C) 2017 En-Pei Lee et al. This is an open access article distributed under the Creative Commons Attribution License, which permits unrestricted use, distribution, and reproduction in any medium, provided the original work is properly cited.

Septic shock and cardiogenic shock are the two most common types of shock in children admitted to pediatric intensive care units (PICUs). The aim of the study was to investigate which hemodynamic variables were associated with mortality in children with shock. We retrospectively analyzed 50 children with shock (37 septic shock cases and 13 cardiogenic shock cases) in the PICU and monitored their hemodynamics using transpulmonary thermodilution from 2003 to 2016. Clinical factors were analyzed between the patients with septic and cardiogenic shock. In addition, hemodynamic parameters associated with mortality were analyzed. The 28 -day mortality was significantly higher in the septic group than in the cardiogenic group $(p=0.016)$. Initially, the parameters of cardiac output and cardiac contractility were higher in the septic group $(p<0.05)$ while the parameters of preload and afterload were all higher in the cardiogenic group $(p<0.05)$. Cardiac index was significantly lower in the nonsurvivors of cardiogenic shock at the time of initial admission and after the first 24 hours (both $p<0.05$ ), while systemic vascular resistance index (SVRI) was significantly lower in the nonsurvivors of septic shock $(p<0.001)$. Therefore, during the first 24 hours after intensive care, SVRI and cardiac index are the most important hemodynamic parameters associated with mortality.

\section{Introduction}

Circulatory shock causes mortality in children and accounts for one-third of cases in intensive care units (ICUs) $[1,2]$. Septic shock and cardiogenic shock are the two most common types accounting for three-fifth and one-fifth of the shock population, respectively, in ICUs $[1,2]$. Some studies reported that the mortality rate was $\sim 40$ to $80 \%$ in septic shock and $60 \%$ in cardiogenic shock $[3,4]$. Delay in the management and recognition of potential clinical symptoms/signs of compensated shock could lead to a high mortality rate [5]. Consequently, timely interventions to maintain an adequate tissue perfusion and oxygenation could significantly decrease the morbidity and mortality in children admitted to ICUs $[6,7]$. Hemodynamic monitoring is essential for the diagnosis and therapeutic management of critically ill patients.
Initially, physical examinations, vital signs, urine output, central venous pressure, and transthoracic echocardiography are often used to evaluate the preload and afterload status and cardiac functions in response to fluid resuscitation [8]. However, numerous studies recently demonstrated the inaccuracy of the methods of assessments for hemodynamic status compared to the objective hemodynamic parameter measurements [9-11]. Advanced hemodynamic monitoring may provide useful and precise data on preload, afterload, cardiac output (CO), cardiac contractility, and severity of pulmonary edema in patients with shock. In addition, assessing the severity of shock guided with an advanced hemodynamic monitoring may assist primary critical care physicians in treating patients and attribute a better clinical outcome.

Transpulmonary thermodilution, such as pulse index continuous $\mathrm{CO}(\mathrm{PiCCO})$, is a less invasive procedure (central 
venous and arterial catheters) and has been widely used in critically ill pediatric patients $[12,13]$. Despite the frequent use of the PiCCO technique in pediatric patients, only few studies compared the hemodynamic parameters between the different types of shock and the chain of alternation between mortality and survival groups after treatment $[14,15]$. In addition, there are insufficient data on what parameters are associated with mortality in critically ill pediatric patients. Therefore, the study aims to compare the parameters of septic and cardiogenic shock using the PiCCO system by analyzing the changes in hemodynamics in the mortality and survival groups. Moreover, we also identified the related parameters in predicting the survival and mortality in the critically ill pediatric patients with septic and cardiogenic shock.

\section{Materials and Methods}

2.1. Patient Population. This retrospective study of children aged 0 to 18 years presenting with shock to the pediatric ICU (PICU) was conducted in a tertiary medical center in Taiwan from 2003 to 2016. The PICU of our hospital was a tertiary ICU with 29 beds and hospitalized patients aged from 1 month to 18 years. The study criteria were uniformly applied to all patients screened in the study, making the study internally standardized based mainly on the international consensus conference, Paris, France, 2006 [16]. The types of shock categorized in mutually exclusive categories in the setting included septic and cardiogenic shock. The study was approved by the Institutional Review Board of Chang Gung Memorial Hospital.

2.2. Study Design. The critically ill children with hemodynamics monitoring via the PiCCO system (PiCCO, Pulsion Medical Systems, Munich, Germany) were included in this study. The transpulmonary thermodilution provided the following: (1) preload parameters: global end-diastolic volume index (GEDVI), intrathoracic blood volume index (ITBVI), and stroke volume variation (SVV); (2) cardiac parameters: $\mathrm{CO}$, cardiac index (CI), and global ejection fraction (GEF); (3) afterload parameters: systemic vascular resistance index (SVRI); and (4) lung parameters: extravascular lung water index (EVLWI) and pulmonary vascular permeability index (PVPI). Information related to the cases of septic and cardiogenic shock included age; sex; cardiac characteristics, such as initial inotropic equivalent, heart rate (beats/min), and mean arterial pressure (MAP; $\mathrm{mmHg}$ ); parameters of the PiCCO system; length of stay in the hospital and PICU; and mortality.

Two sets of measurements were analyzed and compared. Initial parameters were detected within 2 hours of enrollment after the PICU admission. Other data were obtained 24 hours after the critical care under the monitoring of the PiCCO system. Hemodynamic parameters were analyzed between the survivors and nonsurvivors in both the cardiogenic and septic groups. Moreover, we identified the predictors of mortality in the children with cardiogenic and septic shock. The primary outcome was the 28-day mortality rate in the PICU (death from any cause before day 28), and the secondary outcome was the ICU length of stay.
2.3. Measurement of PiCCO Parameters. Three consecutive cold boluses are required for each calibration to obtain the mean measurements [13]. Measurements were performed every 12 hours and whenever any hemodynamic deterioration developed. Data were recorded and exported to the computer using the PiCCO-VoLEF Data Acquisition software (version 6.0; Pulsion Medical Systems) combined with the PiCCO plus device (PC 8100 software version 5.1). The following formula was used:

$$
\Delta \text { SVRI }=\frac{(24 \text {-hour SVRI }- \text { baseline SVRI })}{\text { baseline SVRI }} \times 100
$$

2.4. Statistical Analysis. The Chi-square test, Fisher's exact test, Student's $t$-test, Mann-Whitney $U$ test, and multivariate logistic regression analysis were used where appropriate. In the descriptive analysis, values were presented as means \pm standard deviations (SDs). The difference between the groups was presented as 95\% confidence intervals (CIs). For comparison of dichotomous variables between the groups, the Chi-square test or Fisher's exact test was used. Comparisons of continuous variables between the two groups were performed using the Mann-Whitney $U$ test. Predicted probabilities of mortality and 95\% CIs were calculated using the logistic regression model, and survival was analyzed using the Kaplan-Meier curve. Finally, the receiver operating characteristic (ROC) curve was applied to determine the ideal cut-off values for the hemodynamic parameters for mortality in shock. The test characteristics of the different cut-off values, including sensitivity, specificity, area under the ROC curve (AUC), positive likelihood ratio $\left(\mathrm{LR}^{+}\right)$, and negative likelihood ratio $\left(\mathrm{LR}^{-}\right)$, were also examined.

The AUC, calculated using the trapezoidal rule, was considered a standard measure for the diagnostic value of the parameter. An optimal test result had a value of 1.0, while a useless test result had a value of 0.5 . The $\mathrm{LR}^{+}$and $\mathrm{LR}^{-}$ were calculated for the best cut-off values. The criterion value indicated the value corresponding to the highest accuracy (minimal false negative and false positive results). Statistical significance was set at $p<0.05$. All statistical analyses were performed using the SPSS software (version 22.0; SPSS Inc., Chicago, IL, USA).

\section{Results}

3.1. Demographics of the Children Implanted with the PiCCO Device. During the 13-year study period, 52 children with septic or cardiogenic shock monitored using the PiCCO system were gathered; however, two cases were excluded owing to insufficient data. Therefore, a total of 50 children were reenrolled in our study, with 30 male $(60 \%)$ and 20 female (40\%) patients (Table 1). There were 37 (74\%) cases of septic shock and $13(26 \%)$ cases of cardiogenic shock. The mean age was lower in the cardiogenic group $(9.1 \pm 6.1$ years) than in the septic group (12.2 \pm 4.5 years). The initial cardiac characteristics showed no significant difference between the two groups. However, the 28-day mortality rate was significantly higher in the septic group than in the cardiogenic group (59.5\% versus $15.4 \%, p=0.016$ ) (Figure 1). 
TABLE 1: Demographics of shock cases and initial PiCCO parameters.

\begin{tabular}{|c|c|c|c|}
\hline Variables & $\begin{array}{l}\text { Cardiogenic shock } \\
\quad(n=13)\end{array}$ & $\begin{array}{l}\text { Septic shock } \\
\quad(n=37)\end{array}$ & $p$ value \\
\hline Age (years) & $9.1 \pm 6.1$ & $12.2 \pm 4.5$ & 0.1 \\
\hline Gender & & & 0.43 \\
\hline Male & 9 & 21 & \\
\hline Female & 4 & 16 & \\
\hline \multicolumn{4}{|l|}{ Cardiac characteristics } \\
\hline inotropic equivalent & $30.6 \pm 30.3$ & $46.4 \pm 44.3$ & 0.241 \\
\hline heart rate, beats/min & $131.5 \pm 33.2$ & $138.2 \pm 27.3$ & 0.468 \\
\hline Mean arterial pressure, $\mathrm{mm} \mathrm{Hg}$ & $71.5 \pm 15.1$ & $69.4 \pm 19.2$ & 0.71 \\
\hline \multicolumn{4}{|l|}{ Outcomes } \\
\hline Length of stay (days) & $53.7 \pm 85.7$ & $34.8 \pm 37.9$ & 0.456 \\
\hline ICU stay (days) & $28.1 \pm 32.2$ & $25.1 \pm 32.4$ & 0.779 \\
\hline Mortality & 2 & 22 & 0.016 \\
\hline \multicolumn{4}{|l|}{ PiCCO parameters (Day 1) } \\
\hline \multicolumn{4}{|l|}{ Cardiac output } \\
\hline $\mathrm{CO}, \mathrm{L} / \mathrm{min}$ & $2.68 \pm 0.79$ & $4.22 \pm 1.65$ & $<0.001$ \\
\hline \multicolumn{4}{|l|}{ Cardiac contractility } \\
\hline $\mathrm{CI}\left(\mathrm{L} / \mathrm{min} / \mathrm{m}^{2}\right)$ & $2.84 \pm 1.02$ & $3.75 \pm 1.08$ & 0.011 \\
\hline GEF $(\%)$ & $17.8 \pm 8$ & $27.95 \pm 9.15$ & 0.001 \\
\hline CFI (1/min) & $5.73 \pm 2.54$ & $9.46 \pm 2.76$ & $<0.001$ \\
\hline \multicolumn{4}{|l|}{ Preload parameters } \\
\hline GEDVI $\left(\mathrm{mL} / \mathrm{m}^{2}\right)$ & $519.11 \pm 134.53$ & $420.54 \pm 118.01$ & 0.017 \\
\hline ITBVI $\left(\mathrm{mL} / \mathrm{m}^{2}\right)$ & $648.38 \pm 168.38$ & $525.23 \pm 147.59$ & 0.017 \\
\hline SVV (\%) & $13.84 \pm 5.49$ & $15 \pm 6.5$ & 0.568 \\
\hline \multicolumn{4}{|l|}{ Afterload parameters } \\
\hline SVRI $\left(\mathrm{dyn} * \mathrm{~s} * \mathrm{~cm}^{-5} * \mathrm{~m}^{2}\right)$ & $1936.79 \pm 802.41$ & $1327.34 \pm 705.48$ & 0.013 \\
\hline \multicolumn{4}{|l|}{ Lung parameters } \\
\hline EVLWI $\left(\mathrm{mL} / \mathrm{m}^{2}\right)$ & $18.46 \pm 12.01$ & $14.8 \pm 12.19$ & 0.359 \\
\hline PVPI & $3.99 \pm 2.79$ & $3.88 \pm 2.53$ & 0.898 \\
\hline
\end{tabular}

$\mathrm{ICU}=$ intensive care unit; $\mathrm{CO}=$ cardiac output; $\mathrm{CI}$ = cardiac index; $\mathrm{GEF}$ = global ejection fraction; $\mathrm{CFI}=$ cardiac function index; GEDVI = global end-diastolic volume index; ITBVI = intrathoracic blood volume index; SVV = stroke volume variation; SVRI = systemic vascular resistance index; EVLWI = extravascular lung water index; PVPI = pulmonary vascular permeability index.

3.2. PiCCO Parameters at the Initial Admission and 24 Hours after PICU Admission. As shown in Table 1, the PiCCO parameters of $\mathrm{CO}$ and cardiac contractility, such as $\mathrm{CI}$, GEF, and cardiac function index (CFI), were higher in the septic group than in the cardiogenic group (all $p<0.05$ ). However, the parameters of preload and afterload, including the GEDVI, ITBVI, and SVRI, were higher in the cardiogenic group than in the septic group $(p<0.05)$. The factors between the survivors and nonsurvivors in both groups were identified and are shown in Tables 2 and 3. As shown in Table 2, the MAP was significantly lower in the nonsurvivors than in the survivors in the septic group at the time of PICU admission $(p<0.05)$. However, the CO and CI were significantly lower in the nonsurvivors in the cardiogenic group initially (both $p<0.05$ ). The changes in the PiCCO parameters after treatment for 24 hours are presented in Table 3. The MAP was lower in the nonsurvivors than in the survivors in the septic group $(p<0.001)$. In addition, both the $\mathrm{CO}$ and $\mathrm{CI}$ were lower in the nonsurvivors than in the survivors in the cardiogenic group (both $p<0.05$ ). However, notably, the SVRI was statistically and significantly lower in the nonsurvivors than in the survivors $(901.08 \pm 305.69$ versus $1584.23 \pm 429.63)$ in the septic group $(p<0.001)$.

3.3. Factors Associated with Mortality. The results of the multivariate logistic regression analysis showed that SVRI was an independent predictor of mortality after the 24-hour critical care in the PICU in the septic group (odds ratio [OR], 0.995; 95\% CI, 0.992-0.998, and $p=0.003$ ). Based on the ROC analysis of SVRI in predicting the survivors in the septic group, the AUC was 0.9 (95\% CI, 0.786-1, $p<0.001$ ) (Figure 2). The cut-off values of SVRI in the septic group are shown in Table 4 . We identified SVRI of $1167 \mathrm{dyn} * \mathrm{~s} * \mathrm{~cm}^{-5} * \mathrm{~m}^{2}$ as the appropriate point to predict 


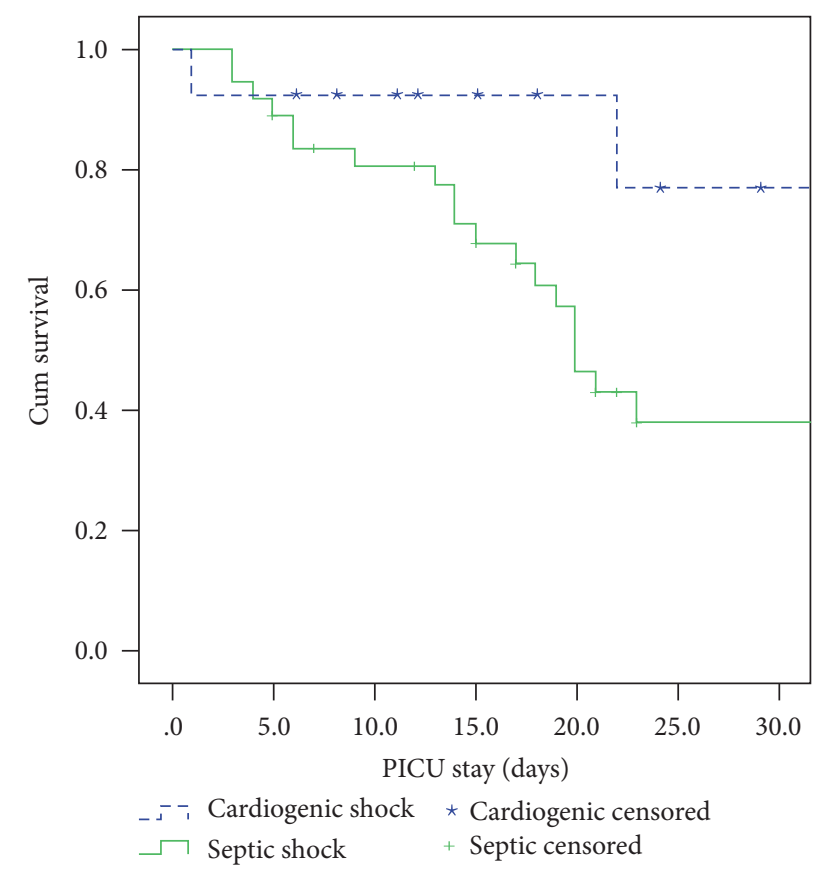

FIGURE 1: Survival rate analysis of children between septic and cardiogenic shock during the first 28 days of PICU stay $(p<0.05)$.

mortality. We also found that the change in SVRI ( $\triangle$ SVRI) was negatively correlated with mortality (OR, $0.974 ; 95 \% \mathrm{CI}$, 0.952-0.997; $p=0.027$ ).

\section{Discussion}

Shock is a major cause of morbidity and mortality in the PICU. In-hospital mortality rates of septic shock are high, ranging between $18 \%$ and $50 \%$ [1]. Mortality increases with the severity of sepsis. Hemodynamic monitoring is essential for the diagnosis and therapeutic management of critically ill patients. In the 13-year retrospective study, we found that SVRI was the most powerful predictor of the 28-day mortality in children with septic shock. There are few studies that demonstrate the importance of SVRI in adults with sepsis [17]; the present study is the first study to identify the importance of SVRI in predicting the mortality in children with septic shock. The SVRI of $1167 \mathrm{dyn} * \mathrm{~s} * \mathrm{~cm}^{-5} * \mathrm{~m}^{2}$ during the first 24 hours after intensive care was the useful predictor of the 28-day mortality. In addition, we found that the change in SVRI $(\Delta$ SVRI) correlated with mortality negatively. In our study, the decreased CI in the children was an independent risk factor for mortality in the cardiogenic group, which was consistent with those of previous studies [18, 19].

A decreased SVRI indicates the expression of injuries in the endothelial layer; endothelial injuries are one of the important pathophysiologies of sepsis [20]. In sepsis, the injured endothelial cells could increase the secretions of reactive oxidants, lytic enzymes, prostacyclin, lipopolysaccharide, vasoactive substances, such as endothelin, plateletderived growth factor, and the most important substanceoverproduction of nitric oxide (NO) [20, 21]. Increasing

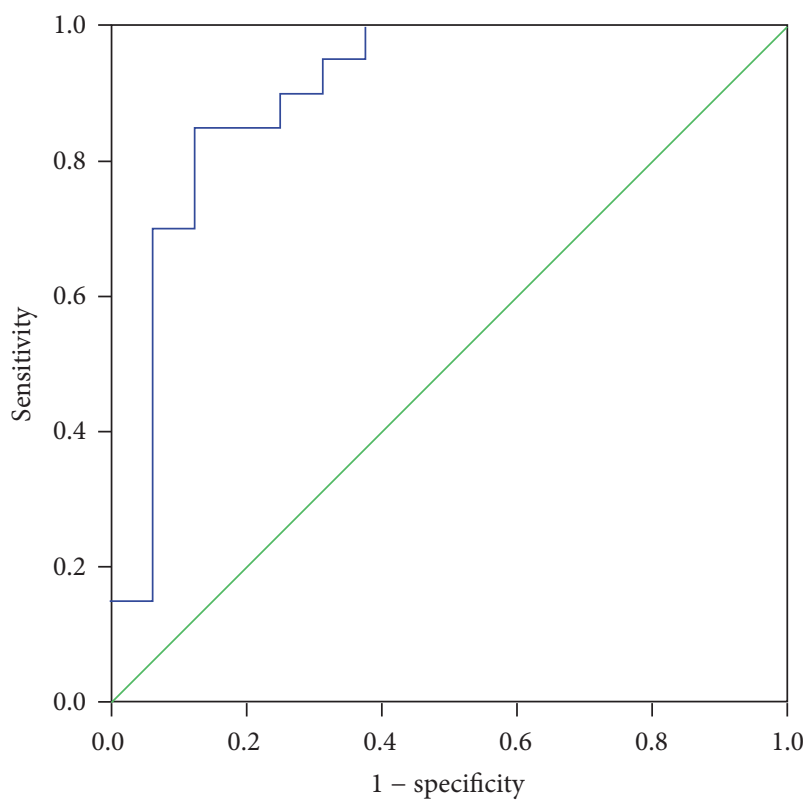

FIGURE 2: Receiver operator characteristic analysis for SVR in predicting mortality in septic shock after the 24 hours of admission to the PICU.

NO synthesis by injured endothelial cells would damage the cerebral autonomic centers, which would further reduce the vascular reactivity to vasoconstrictors, causing a refractory hypotension [22, 23]. Another important factor causing hypotension in sepsis is the decreased compensatory secretion of vasopressin, which may be caused by impairing the baroreflex-mediated secretion [24]. Therefore, hypotension due to vasodilation, especially from endothelial injuries, may be the critical cause of circulatory malfunction in sepsis.

Although vasodilation induced by endothelial injuries may be the predictor of mortality in septic patients reported in some studies [20], the clinical application of SVRI has not been established in children. We estimated the association between the hemodynamic variables and clinical outcomes during the first 24 hours after intensive care because the therapeutic treatment during the early phase of shock could be crucial for survival $[19,25,26]$. The study demonstrated that the decreased value of SVRI after the 24-hour intensive care may serve as the early predictor of prognosis in children with sepsis, which is consistent with the results of a previous study in adults [17]. Several studies reported that the severity of pulmonary edema evaluated using the EVLWI and PVPI was the independent risk factor for mortality in sepsis [15, 18 ,27]. However, although the nonsurvivors in our study had higher EVLWI and PVPI levels than that of the survivors, no significant difference was noted in the first 24 hours after the treatment. The difference may be that other studies analyzed the EVLWI at the maximum value and often developed 72 hours after intensive care, which is compatible with the clinical course of severe pulmonary edema commonly developing after 72 hours of intensive care [18, 27, 28].

On the other hand, CI was the independent risk factor for mortality in the pediatric cardiogenic shock in our study, 
TABLE 2: Initial PiCCO parameters between survivors and nonsurvivors in children with cardiogenic shock and septic shock at the time of admission to the PICU.

\begin{tabular}{|c|c|c|c|c|c|c|}
\hline \multirow{2}{*}{ Characteristics } & \multicolumn{3}{|c|}{ Cardiogenic Shock } & \multicolumn{3}{|c|}{ Septic shock } \\
\hline & Death $(n=3)$ & Survival $(n=11)$ & $p$ value & Death $(n=22)$ & Survival $(n=15)$ & $p$ value \\
\hline Age (years) & $8.7 \pm 10.89$ & $9.15 \pm 5.69$ & 0.927 & $12.62 \pm 4.3$ & $11.53 \pm 4.81$ & 0.476 \\
\hline Gender & & & 0.522 & & & 0.729 \\
\hline Male & 1 & 8 & & 13 & 8 & \\
\hline Female & 1 & 3 & & 9 & 7 & \\
\hline LOS in ICU & $11.5 \pm 14.85$ & $31.01 \pm 34.04$ & 0.453 & $24.05 \pm 38.43$ & $26.73 \pm 22.02$ & 0.808 \\
\hline \multicolumn{7}{|l|}{ Cardiac characteristics } \\
\hline inotropic equivalent & $63.75 \pm 76.91$ & $24.55 \pm 17.04$ & 0.598 & $56.11 \pm 54.33$ & $32.17 \pm 15.93$ & 0.107 \\
\hline heart rate (beats/min) & $121.75 \pm 32.17$ & $132.23 \pm 34.63$ & 0.673 & $137.83 \pm 28.51$ & $138.94 \pm 26.38$ & 0.906 \\
\hline Mean arterial pressure (mm Hg) & $67.34 \pm 26.4$ & $72.3 \pm 14.09$ & 0.687 & $64.24 \pm 17.73$ & $76.87 \pm 19.4$ & 0.048 \\
\hline \multicolumn{7}{|l|}{ PiCCO parameters } \\
\hline \multicolumn{7}{|l|}{ Cardiac output } \\
\hline $\mathrm{CO}(\mathrm{L} / \mathrm{min})$ & $1.6 \pm 0.06$ & $2.88 \pm 0.69$ & 0.028 & $4.31 \pm 1.59$ & $4.09 \pm 1.76$ & 0.705 \\
\hline \multicolumn{7}{|l|}{ Cardiac contractility } \\
\hline $\mathrm{CI}\left(\mathrm{L} / \mathrm{min} / \mathrm{m}^{2}\right)$ & $1.34 \pm 0.09$ & $2.93 \pm 0.98$ & 0.049 & $3.68 \pm 0.93$ & $3.86 \pm 1.29$ & 0.626 \\
\hline GEF (\%) & $15.25 \pm 4.59$ & $18.27 \pm 8.55$ & 0.645 & $27.49 \pm 10.26$ & $28.56 \pm 7.72$ & 0.739 \\
\hline CFI (1/min) & $4.48 \pm 2.93$ & $5.91 \pm 2.58$ & 0.584 & $9.01 \pm 2.59$ & $10.08 \pm 2.95$ & 0.263 \\
\hline \multicolumn{7}{|l|}{ Preload parameters } \\
\hline GEDVI $\left(\mathrm{mL} / \mathrm{m}^{2}\right)$ & $458.75 \pm 51.97$ & $530.08 \pm 143.48$ & 0.514 & $424.52 \pm 107.12$ & $415.23 \pm 134.88$ & 0.822 \\
\hline ITBVI $\left(\mathrm{mL} / \mathrm{m}^{2}\right)$ & $572.41 \pm 65.17$ & $662.19 \pm 179.54$ & 0.512 & $530.18 \pm 134.03$ & $518.64 \pm 168.63$ & 0.823 \\
\hline SVV (\%) & $12.58 \pm 0.12$ & $14.07 \pm 5.98$ & 0.741 & $15.54 \pm 7.23$ & $14.21 \pm 5.39$ & 0.55 \\
\hline \multicolumn{7}{|l|}{ Afterload parameters } \\
\hline SVRI, dyn $* \mathrm{~s} * \mathrm{~cm}^{-5} * \mathrm{~m}^{2}$ & $1794.4 \pm 219.08$ & $1962.67 \pm 873.53$ & 0.798 & $1196.75 \pm 509.52$ & $1510.17 \pm 901.11$ & 0.193 \\
\hline \multicolumn{7}{|l|}{ Lung parameters } \\
\hline EVLWI, $\mathrm{mL} / \mathrm{m}^{2}$ & $18.16 \pm 3.06$ & $18.52 \pm 13.18$ & 0.97 & $15.34 \pm 13.51$ & $14.09 \pm 10.58$ & 0.769 \\
\hline PVPI & $4.02 \pm 2$ & $3.98 \pm 2.99$ & 0.99 & $3.99 \pm 2.84$ & $3.74 \pm 2.12$ & 0.774 \\
\hline
\end{tabular}

ICU = intensive care unit; $\mathrm{CO}$ = cardiac output; $\mathrm{CI}$ = cardiac index; $\mathrm{GEF}$ = global ejection fraction; $\mathrm{CFI}=$ cardiac function index; GEDVI = global end-diastolic volume index; ITBVI = intrathoracic blood volume index; SVV = stroke volume variation; SVRI = systemic vascular resistance index; EVLWI = extravascular lung water index; PVPI = pulmonary vascular permeability index.

and the results were consistent with those of previous studies in adults $[19,26,29]$. According to the pathophysiology, CI may be related to the base deficit. Our study observed that a decreased CI in the first 24 hours after intensive care could reflect the failure of hemodynamic interventions in nonsurvivors. Although only two nonsurvivors were included in our analysis, both cases had the lowest CIs among the cases of cardiogenic shock.

In conclusion, SVRI and CI are the most important hemodynamic parameters associated with the 28-day mortality in children with septic shock and cardiogenic shock, respectively, during the first 24 hours after intensive care. Most importantly, we determined the SVRI of $1167 \mathrm{dyn} * \mathrm{~s}$ $* \mathrm{~cm}^{-5} * \mathrm{~m}^{2}$ as the best appropriate predictor of mortality after 24-hour intensive care interventions.

\section{Abbreviations}

PICU: Pediatric intensive care unit

PiCCO: Pulse index continuous cardiac output

GEDVI: Global end-diastolic volume index
ITBVI: Intrathoracic blood volume index

SVV: Stroke volume variation

CO: $\quad$ Cardiac output

CI: $\quad$ Cardiac index

GEF: Global ejection fraction

SVRI: Systemic vascular resistance index

EVLWI: Extravascular lung water index

PVPI: Pulmonary vascular permeability index

LOS: Length of stay

SD: $\quad$ Standard deviation

CIs: Confidence intervals.

\section{Ethical Approval}

The study protocol was approved by the Institution Review Board and ethics committee of Chang Gung Memorial hospital.

\section{Conflicts of Interest}

The authors declare that they have no conflicts of interest. 
TABLE 3: The PiCCO parameters between survivors and nonsurvivors after 24 hours of setting up the PiCCO.

\begin{tabular}{|c|c|c|c|c|c|c|}
\hline \multirow{2}{*}{ Variables } & \multicolumn{3}{|c|}{ Cardiogenic shock } & \multicolumn{3}{|c|}{ Septic shock } \\
\hline & Death $(n=3)$ & Survival $(n=11)$ & $p$ value & Death $(n=22)$ & Survival $(n=15)$ & $p$ value \\
\hline \multicolumn{7}{|l|}{ Cardiac characteristics } \\
\hline heart rate (beats/min) & $120 \pm 33.9$ & $137.3 \pm 39.2$ & 0.572 & $139.18 \pm 26.26$ & $125.64 \pm 32.45$ & 0.169 \\
\hline Mean arterial pressure $(\mathrm{mm} \mathrm{Hg})$ & $68.5 \pm 23.3$ & $79.9 \pm 12$ & 0.292 & $59.8 \pm 14.84$ & $84.13 \pm 19.19$ & $<0.001$ \\
\hline \multicolumn{7}{|l|}{ PiCCO parameters } \\
\hline \multicolumn{7}{|l|}{ Cardiac output } \\
\hline $\mathrm{CO}(\mathrm{L} / \mathrm{min})$ & $1.53 \pm 0.01$ & $3.57 \pm 0.97$ & $<0.001$ & $5.01 \pm 1.59$ & $3.99 \pm 1.55$ & 0.062 \\
\hline \multicolumn{7}{|l|}{ Cardiac contractility } \\
\hline $\mathrm{CI}\left(\mathrm{L} / \mathrm{min} / \mathrm{m}^{2}\right)$ & $1.33 \pm 0.11$ & $3.59 \pm 1.32$ & 0.039 & $4.23 \pm 0.92$ & $3.77 \pm 1.01$ & 0.166 \\
\hline GEF $(\%)$ & $15 \pm 4.24$ & $19.4 \pm 7.56$ & 0.451 & $30.93 \pm 11.29$ & $30.02 \pm 11.46$ & 0.812 \\
\hline CFI (1/min) & $4.65 \pm 2.76$ & $6.56 \pm 2.45$ & 0.336 & $9.69 \pm 3.34$ & $9.09 \pm 2.92$ & 0.574 \\
\hline \multicolumn{7}{|l|}{ Preload parameters } \\
\hline $\operatorname{GEDVI}\left(\mathrm{mL} / \mathrm{m}^{2}\right)$ & $452.5 \pm 47.38$ & $572.06 \pm 106.39$ & 0.157 & $457.82 \pm 140.55$ & $449.44 \pm 156.64$ & 0.867 \\
\hline ITBVI $\left(\mathrm{mL} / \mathrm{m}^{2}\right)$ & $565 \pm 59.39$ & $714.82 \pm 132.98$ & 0.156 & $571.84 \pm 175.74$ & $561.3 \pm 195.83$ & 0.866 \\
\hline SVV $(\%)$ & $11.25 \pm 3.18$ & $13.48 \pm 5.72$ & 0.61 & $15 \pm 5.97$ & $11.3 \pm 5.87$ & 0.068 \\
\hline \multicolumn{7}{|l|}{ Afterload parameters } \\
\hline SVRI, $\left(\mathrm{dyn} * \mathrm{~s} * \mathrm{~cm}^{-5} * \mathrm{~m}^{2}\right)$ & $1742 \pm 270.11$ & $1664.48 \pm 469.75$ & 0.829 & $901.08 \pm 305.69$ & $1584.23 \pm 429.63$ & $<0.001$ \\
\hline \multicolumn{7}{|l|}{ Lung parameters } \\
\hline $\operatorname{EVLWI}\left(\mathrm{mL} / \mathrm{m}^{2}\right)$ & $16.5 \pm 2.12$ & $17 \pm 11.63$ & 0.954 & $16.5 \pm 13.85$ & $11.88 \pm 6.53$ & 0.229 \\
\hline PVPI & $3.65 \pm 1.63$ & $3.06 \pm 1.52$ & 0.626 & $4.07 \pm 2.8$ & $3.09 \pm 1.36$ & 0.212 \\
\hline
\end{tabular}

ICU = intensive care unit; $\mathrm{CO}$ = cardiac output; $\mathrm{CI}$ = cardiac index; $\mathrm{GEF}$ = global ejection fraction; $\mathrm{CFI}$ = cardiac function index; GEDVI = global end-diastolic volume index; ITBVI = intrathoracic blood volume index; SVV = stroke volume variation; SVRI = systemic vascular resistance index; EVLWI = extravascular lung water index; PVPI = pulmonary vascular permeability index.

TABLE 4: Predictive power of SVRI for different cut-off points in noncardiogenic group.

\begin{tabular}{|c|c|c|c|c|c|}
\hline SVRI value & Sensitivity & Specificity & $\mathrm{LR}^{+}$ & $\mathrm{LR}^{-}$ & Youden index \\
\hline 533 & 0.15 & 1.0 & - & 0.85 & 0.15 \\
\hline 591 & 0.15 & 0.938 & 2.4 & 0.907 & 0.0875 \\
\hline 1093 & 0.7 & 0.937 & 11.2 & 0.32 & 0.6375 \\
\hline 1115 & 0.7 & 0.875 & 5.6 & 0.347 & 0.575 \\
\hline $1167^{*}$ & 0.85 & 0.875 & 6.8 & 0.171 & 0.725 \\
\hline 1351 & 0.85 & 0.75 & 3.4 & 0.2 & 0.6 \\
\hline 1362 & 0.9 & 0.75 & 3.6 & 0.133 & 0.65 \\
\hline 1371 & 0.9 & 0.687 & 2.88 & 0.145 & 0.5875 \\
\hline 1394 & 0.95 & 0.687 & 3.04 & 0.073 & 0.6375 \\
\hline 1460 & 0.95 & 0.625 & 2.533 & 0.08 & 0.575 \\
\hline 1531 & 1.0 & 0.625 & 2.667 & 0 & 0.625 \\
\hline
\end{tabular}

$\mathrm{LR}^{+}$: likelihood ratio for a positive test; $\mathrm{LR}^{-}$: likelihood ratio for a negative test; $*$ : best cut-off point.

\section{Authors' Contributions}

En-Pei Lee and Shao-Hsuan Hsia contributed equally to the study. Shao-Hsuan Hsia and Jainn-Jim Lin participated in data analysis. Oi-Wa Chan and Chia-Ying Lin gathered the data. En-Pei Lee, Shao-Hsuan Hsia, Jung Lee, and Han-Ping Wu drafted the manuscript, with all authors revising it critically for intellectual content. All authors have read and approved the final version of this manuscript. En-Pei Lee and Shao-Hsuan Hsia contributed equally to this work.

\section{Acknowledgments}

The authors thank the statistician in Chang Gung Memorial Hospital for completing the statistical analysis. 


\section{References}

[1] M.-Y. Huang, C.-Y. Chen, J.-H. Chien et al., "Serum procalcitonin and procalcitonin clearance as a prognostic biomarker in patients with severe sepsis and septic shock," BioMed Research International, vol. 2016, Article ID 1758501, 5 pages, 2016.

[2] D. De Backer, P. Biston, J. Devriendt et al., "Comparison of dopamine and norepinephrine in the treatment of shock," New England Journal of Medicine, vol. 362, no. 9, pp. 779-789, 2010.

[3] I. Jawad, I. Lukšić, and S. B. Rafnsson, "Assessing available information on the burden of sepsis: global estimates of incidence, prevalence and mortality," Journal of Global Health, vol. 2, no. 1, Article ID 010404, 2012.

[4] H. H. Awad, F. A. Anderson Jr., J. M. Gore, S. G. Goodman, and R. J. Goldberg, "Cardiogenic shock complicating acute coronary syndromes: insights from the Global Registry of Acute Coronary Events," American Heart Journal, vol. 163, no. 6, pp. 963-971, 2012.

[5] A. Kumar, D. Roberts, K. E. Wood et al., "Duration of hypotension before initiation of effective antimicrobial therapy is the critical determinant of survival in human septic shock," Critical Care Medicine, vol. 34, no. 6, pp. 1589-1596, 2006.

[6] G. Y. Larsen, N. Mecham, and R. Greenberg, "An emergency department septic shock protocol and care guideline for children initiated at triage," Pediatrics, vol. 127, no. 6, pp. e1585e1592, 2011.

[7] A. T. Cruz, A. M. Perry, E. A. Williams, J. M. Graf, E. R. Wuestner, and B. Patel, "Implementation of goal-directed therapy for children with suspected sepsis in the emergency department," Pediatrics, vol. 127, no. 3, pp. e758-e766, 2011.

[8] S. M. Tibby, M. Hatherill, and I. A. Murdoch, "Use of transesophageal Doppler ultrasonography in ventilated pediatric patients: derivation of cardiac output," Critical Care Medicine, vol. 28, no. 6, pp. 2045-2050, 2000.

[9] J. Perny, A. Kimmoun, P. Perez, and B. Levy, "Evaluation of cardiac function index as measured by transpulmonary thermodilution as an indicator of left ventricular ejection fraction in cardiogenic shock," BioMed Research International, vol. 2014, Article ID 598029, 7 pages, 2014.

[10] A.-T. Lobos, S. Lee, and K. Menon, "Capillary refill time and cardiac output in children undergoing cardiac catheterization," Pediatric Critical Care Medicine, vol. 13, no. 2, pp. 136-140, 2012.

[11] S. M. Tibby, M. Hatherill, A. Durward, and I. A. Murdoch, "Are transoesophageal Doppler parameters a reliable guide to paediatric haemodynamic status and fluid management?" Intensive Care Medicine, vol. 27, no. 1, pp. 201-205, 2001.

[12] S. Tibby, "Transpulmonary thermodilution: finally, a gold standard for pediatric cardiac output measurement," Pediatric Critical Care Medicine, vol. 9, no. 3, pp. 341-342, 2008.

[13] X. Monnet, R. Persichini, M. Ktari, M. Jozwiak, C. Richard, and J.-L. Teboul, "Precision of the transpulmonary thermodilution measurements," Critical Care, vol. 15, article R204, 2011.

[14] M. Jozwiak, J.-L. Teboul, and X. Monnet, "Extravascular lung water in critical care: recent advances and clinical applications," Annals of Intensive Care, vol. 5, no. 1, article 38, 2015.

[15] W. Chen, X. Zang, S. Niu et al., "Early predictive value of hemodynamic parameters during fluid resuscitation in patients with sepsis shock," Zhonghua wei zhong bing ji jiu yi xue, vol. 27, no. 1, pp. 43-47, 2015.

[16] M. Antonelli, M. Levy, P. J. D. Andrews et al., "Hemodynamic monitoring in shock and implications for management.
International Consensus Conference, Paris, France, 27-28 April 2006.", Intensive care medicine, vol. 33, no. 4, pp. 575-590, 2007.

[17] L. Metrangolo, M. Fiorillo, G. Friedman et al., "Early hemodynamic course of septic shock," Critical Care Medicine, vol. 23, no. 12, pp. 1971-1975, 1995.

[18] Z. Zhang, B. Lu, and H. Ni, "Prognostic value of extravascular lung water index in critically ill patients: a systematic review of the literature," Journal of Critical Care, vol. 27, no. 4, pp. 420.e1420.e8, 2012.

[19] C. Torgersen, C. A. Schmittinger, S. Wagner et al., "Hemodynamic variables and mortality in cardiogenic shock: a retrospective cohort study," Critical Care, vol. 13, article R157, 2009.

[20] J. Boisramé-Helms, H. Kremer, V. Schini-Kerth, and F. Meziani, "Endothelial dysfunction in sepsis," Current Vascular Pharmacology, vol. 11, no. 2, pp. 150-160, 2013.

[21] J. Vincent, H. Zhang, C. Szabo, and J. Preiser, "Effects of nitric oxide in septic shock," American Journal of Respiratory and Critical Care Medicine, vol. 161, no. 6, pp. 1781-1785, 2000.

[22] M. A. Titheradge, "Nitric oxide in septic shock," Biochimica et Biophysica Acta (BBA)_Bioenergetics, vol. 1411, no. 2-3, pp. 437455, 1999.

[23] T. Sharshar, F. Gray, G. L. De La Grandmaison et al., "Apoptosis of neurons in cardiovascular autonomic centres triggered by inducible nitric oxide synthase after death from septic shock," Lancet, vol. 362, no. 9398, pp. 1799-1805, 2003.

[24] J. A. Russell, "Bench-to-bedside review: vasopressin in the management of septic shock," Critical Care, vol. 15, article 226, 2011.

[25] E. Rivers, B. Nguyen, S. Havstad et al., "Early goal-directed therapy in the treatment of severe sepsis and septic shock," The New England Journal of Medicine, vol. 345, pp. 1368-1377, 2001.

[26] R. Fincke, J. Hochman, and A. Lowe, "Cardiac power is the strongest hemodynamic correlate of mortality in cardiogenic shock: a report from the shock trial registry," ACC Current Journal Review, vol. 13, no. 11, p. 49, 2004.

[27] G. S. Martin, S. Eaton, M. Mealer, and M. Moss, "Extravascular lung water in patients with severe sepsis: a prospective cohort study," Critical Care, vol. 9, no. 2, pp. R74-R82, 2005.

[28] S. G. Sakka, M. Klein, K. Reinhart, and A. Meier-Hellmann, "Prognostic value of extravascular lung water in critically III patients," Chest, vol. 122, no. 6, pp. 2080-2086, 2002.

[29] H. R. Reynolds and J. S. Hochman, "Cardiogenic shock: current concepts and improving outcomes," Circulation, vol. 117, no. 5, pp. 686-697, 2008. 


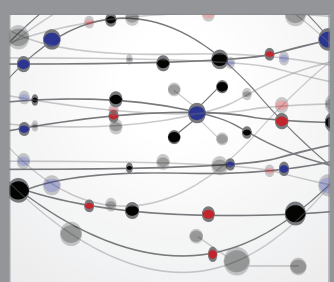

The Scientific World Journal
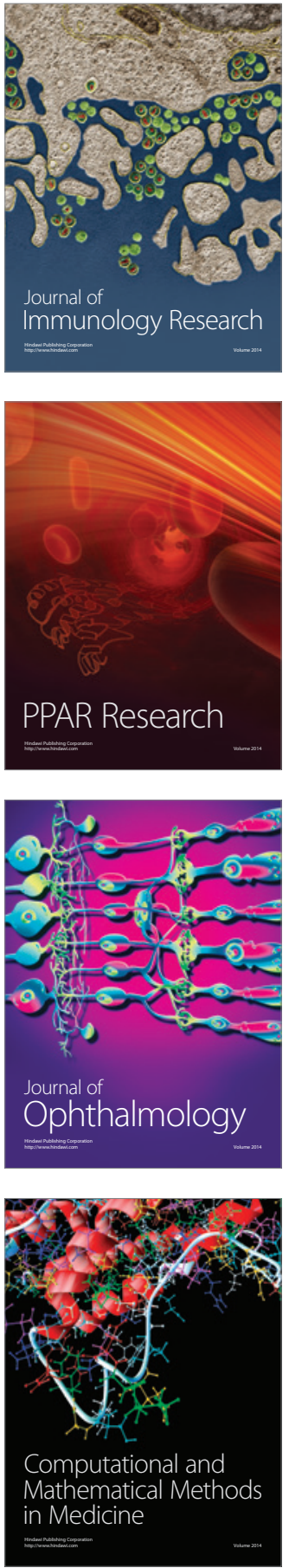

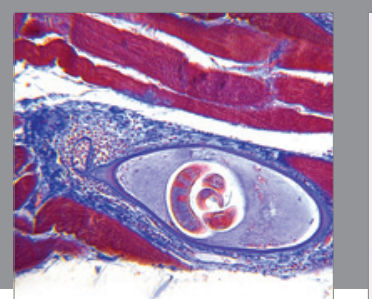

Gastroenterology Research and Practice
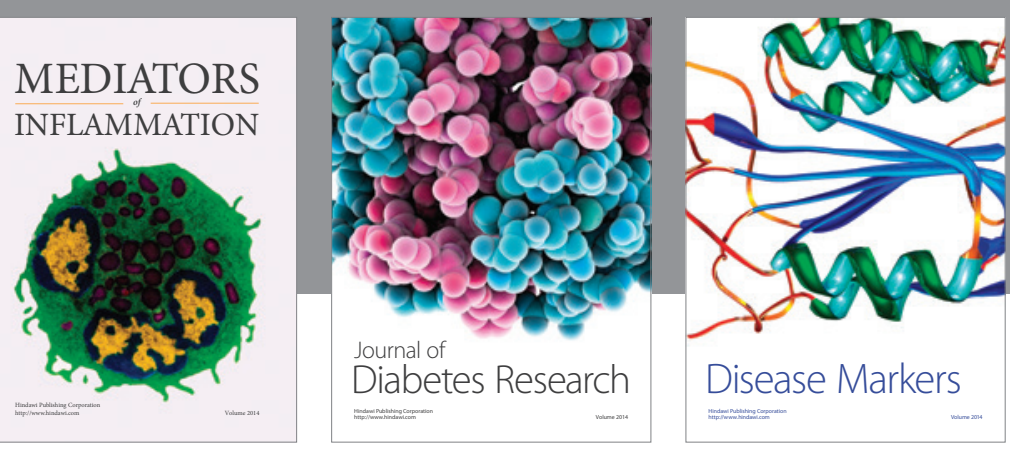

Disease Markers

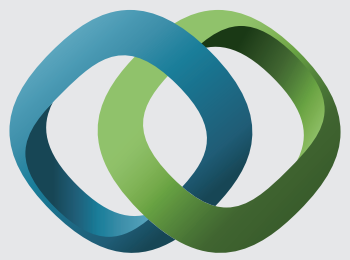

\section{Hindawi}

Submit your manuscripts at

https://www.hindawi.com
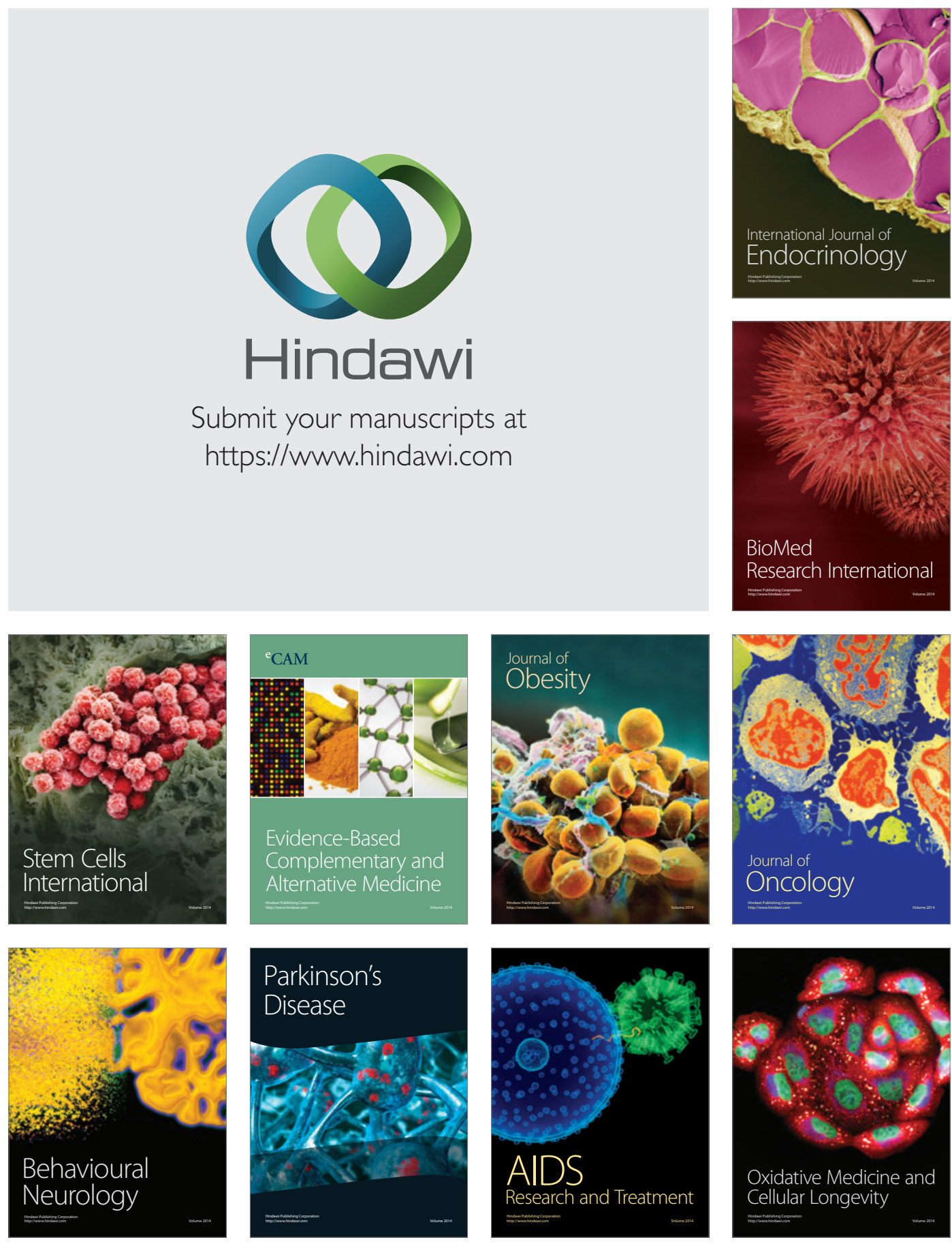